\title{
Glassy Relaxation without Freezing in a Random Dipolar-Coupled Ising Magnet
}

\author{
D. H. Reich and T. F. Rosenbaum \\ The James Franck Institute and the Department of Physics, The University of Chicago, \\ Chicago, Illinois 60637 \\ and \\ G. Aeppli
}

AT\& T Bell Laboratories, Murray Hill, New Jersey 07974

(Received 1 May 1987)

\begin{abstract}
We have measured the magnetic susceptibility, $\chi^{\prime}+i \chi^{\prime \prime}$, of the dilute dipolar-coupled Ising magnet $\mathrm{LiHo}_{0.045} \mathrm{Y}_{0.955} \mathrm{~F}_{4}$ over six decades of frequency from $0.02 \mathrm{~Hz}$ to $20 \mathrm{kHz}$. The system behaves as an ideal relaxational glass with Arrhenius behavior in temperature of the peak in $\chi^{\prime \prime}$. Scaling data from $T=100$ $\mathrm{mK}$ to $T=300 \mathrm{mK}$ by the peak in $\chi^{\prime \prime}$ shows an enhanced low-frequency response at high temperatures, in contrast to expectations for spin-glasses and random-field magnets.
\end{abstract}

PACS numbers: $75.40 . \mathrm{Gb}, 75.50 . \mathrm{Kj}$

Most classical spin systems undergo freezing (or ordering) as the temperature $T$ is reduced. The few exceptions, notably the triangular antiferromagnet ${ }^{1}$ and the random antiferromagnet, ${ }^{2}$ are theoretical constructs which have yet to be realized experimentally. In this paper, we present experimental evidence that an interacting classical spin system need not freeze as the temperature approaches zero. Specially, our ac susceptibility measurements indicate that for $\mathrm{LiHo}_{0.045} \mathrm{Y}_{0.955} \mathrm{~F}_{4}$ the population of high-energy barriers to spin relaxation actually decreases with $T$. For comparison, recall that the opposite occurs for the spin-glass and random-field problems, leading to the anomalously slow relaxation which has been a primary theme of recent research on disordered systems. $^{3}$

As described in an earlier study, ${ }^{4} \mathrm{LiHo}_{x} \mathrm{Y}_{1-x} \mathrm{~F}_{4}$ is a dilute, insulating, dipolar-coupled Ising system. For $x$ larger than, at most, 0.46 , the members of this isostructural series order ferromagnetically at $T_{c}=x T_{c}(x=1)$ with $T_{c}=1.53 \mathrm{~K}$ for $\mathrm{LiHoF}_{4}$. On the other hand, for $x<0.17$, no evidence for ferromagnetic order above $T$ $=10 \mathrm{mK}$ has been found. The $x=0.045$ single crystal studied here has dimensions $0.95 \times 0.52 \times 0.52 \mathrm{~cm}^{3}$, with the Ising $(c)$ axis parallel to its length. We used an inductance bridge technique to measure the frequencydependent susceptibility $\chi(\omega)=\chi^{\prime}(\omega)+i \chi^{\prime \prime}(\omega)$ at eight temperatures between 90 and $300 \mathrm{mK}$. The bridge contained two identical mutual inductances bolted to the mixing chamber of a helium dilution refrigerator, with the secondary (pickup) coils wired in opposition. The secondaries each had 2750 turns of 40-gauge $\mathrm{Cu}$ wire wound in 25 layers around a Mylar form of the same dimensions as the sample. The primaries had 1460 turns of 36-gauge $\mathrm{Cu}$ wire in four layers and were $5 \mathrm{~cm}$ long to provide a magnetic field $H<0.04$ Oe that varied by less than $1 \%$ over the length of the sample. With the sample in one arm of the bridge, the empty arm served to cancel out the signal from the coils themselves and the voltage measured was proportional to $\chi(\omega)$. The primaries were driven with a constant current, measured across a metal film resistor in series. $\chi^{\prime \prime}(\omega)$ was taken to be the component of the signal in phase with the current, and $\chi^{\prime}(\omega)$ as the out-of-phase component. We measured the magnetic response from $0.2 \mathrm{~Hz}$ to $20 \mathrm{kHz}$ with a PAR model 124A lock-in amplifier, and from 0.02 to $2 \mathrm{~Hz}$ with a combination Hewlett-Packard model 3325A frequency synthesizer, computer-based digital lock-in technique. ${ }^{5}$

Figure 1 shows the real and imaginary parts of the

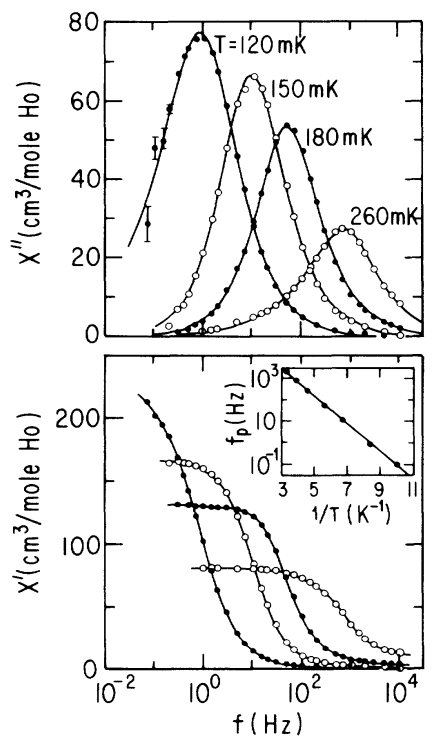

FIG. 1. Real $\left(\chi^{\prime}\right)$ and imaginary $\left(\chi^{\prime \prime}\right)$ parts of the magnetic susceptibility vs frequency $f$ for $\mathrm{LiHo}_{0.045} \mathrm{Y}_{0.955} \mathrm{~F}_{4}$. The solid lines are fits by Eqs. (4) and (5) in the text. Inset: Arrhenius behavior [Eq. (1)] of the peak frequency $f_{p}$ of $\chi^{\prime \prime}$ with temperature $T$. 
susceptibility over six decades in frequency at four different temperatures. At each $T, \chi^{\prime \prime}(\omega)$ has a wellresolved maximum which coincides roughly with the inflection point in $\chi^{\prime}(\omega)$. The data include a demagnetization correction, where we have approximated the shape of the sample by a prolate ellipsoid of revolution ${ }^{6}$ with a ratio of major to minor axes of 1.8. The solid lines are nonlinear least-squares fits by the form given by Eqs. (4) and (5), discussed below.

As shown in the inset to Fig. 1, the characteristic peak frequency $f_{p}$, defined by the maximum in $\chi^{\prime \prime}$, obeys an Arrhenius law

$$
f_{p}=f_{0} \exp \left(-E_{B} / k T\right),
$$

where $f_{0}=2.0 \times 10^{5} \mathrm{~Hz}$ and $E_{B} / k=1.46 \mathrm{~K}$; observe that lowering $T$ from 300 to $90 \mathrm{mK}$ reduces $f_{p}$ by 4 orders of magnitude. In order to determine whether a single process, namely that giving rise to $f_{p}$, can account for the entire magnetic response of $\mathrm{LiHo}_{0.045} \mathrm{Y}_{0.955} \mathrm{~F}_{4}$, we have compared the scaled susceptibilities, $\chi^{\prime \prime}\left(f / f_{p}\right) / \chi^{\prime \prime}\left(f_{p}\right)$, for five different temperatures in Fig. 2. It appears that for $f>f_{p} / 2$, the scaled response functions are indistinguishable. On the other hand, for $f<f_{p} / 10$, substantial differences exist: At the higher temperatures, there is a larger low-frequency response. In other words, with decreasing $T, \chi^{\prime \prime}(\omega)$ becomes narrower in the sense that it loses weight at low (scaled) frequencies. We note that this unusual low-frequency behavior is completely insensitive to our choice of demagnetization factor because of the scaling by the peak frequency.

Perhaps the simplest magnetic response function is the Debye form ${ }^{7}$ :

$$
\chi(\omega)=\chi_{0} /(1-i \omega \tau)
$$

which, according to the fluctuation-dissipation theorem, corresponds to a magnetization autocorrelation function $\langle M(t) M(0)\rangle \sim \exp [-(t / \tau)]$ for $k T \gg \hbar / \tau$. The Debye

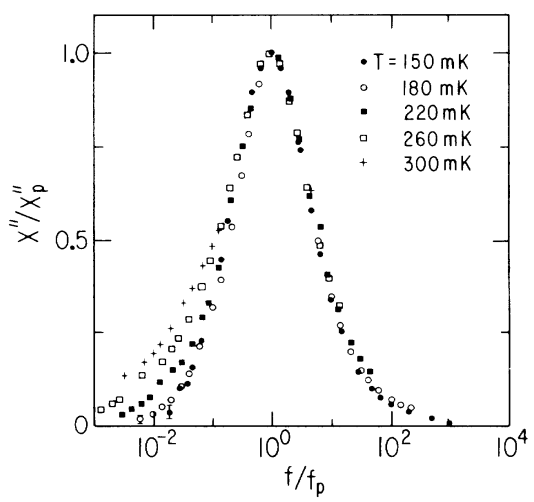

FIG. 2. Imaginary part $\chi^{\prime \prime}$ of the susceptibility scaled by peak frequency $f_{p}$ at five different temperatures. There is enhanced low- (scaled) frequency response at high temperatures, unlike almost all random magnetic systems. form gives a full width at half maximum (FWHM) in $\chi^{\prime \prime}(\omega)$ of 1.14 decades in frequency. The response of $\mathrm{LiHo}_{0.045} \mathrm{Y}_{0.955} \mathrm{~F}_{4}$ plotted in Fig. 1 has a FWHM approaching two decades in frequency; hence, it cannot be described by a single relaxation time $\tau$. We have tried as well the standard asymmetric Cole-Davidson form, ${ }^{8}$

$$
\chi(\omega)=\chi_{0} /(1-i \omega \tau)^{\beta},
$$

which is shown for $T=220 \mathrm{mK}$ by the dotted line in Fig. 3 with the best-fit value $\beta=0.54$. This function deviates greatly from the data at both low and high frequencies. Better results are obtained when we assume the form associated with a flat distribution of relaxation times,

$$
\chi(\omega)=\int_{0}^{\infty} \rho(\tau) \chi_{\tau}(\omega) d \tau
$$

where $\chi_{\tau}(\omega)$ is given by Eq. (2) and $\rho_{\tau}(\tau)=0$ for $\tau>\tau_{c}$. and $\tau_{c}{ }^{-1}$ for $\tau<\tau_{c}$. The fits obtained are adequate at low temperatures, but fail to duplicate the low-frequency behavior at higher $T$ (dashed line in Fig. 3), a problem symptomatic of Lorentzian and Gaussian forms for $\rho(\tau)$ as well.

In view of the relative success of Eq. (4) and because the deviations occur at small $\omega$, which correspond to long times, we have added a large- $\tau$ tail to the square distribution used in Eq. (4), viz.,

$$
\rho_{\tau}(\tau)= \begin{cases}(1-a) / \tau_{c} & \text { for } \tau<\tau_{c}, \\ a y \tau_{c}^{y} / \tau^{y+1} & \text { for } \tau>\tau_{c} .\end{cases}
$$

Substitution of (5) into (4) yields a function which provides an excellent description of the data at all temperatures. The real and imaginary parts of $\chi(\omega)$ were fitted separately; the two fits yielded the same values for $a, y$, $\chi_{0}$, and $\tau_{c}$ to within $10 \%$. Over the range of tempera-

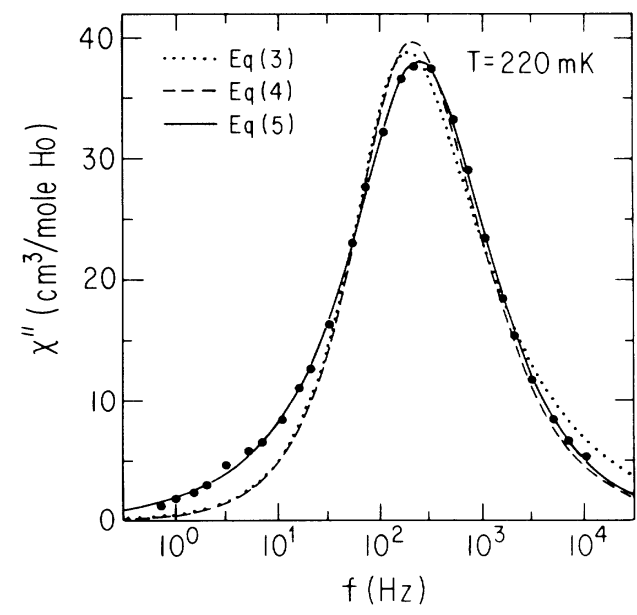

FIG. 3. Comparison of various fitting forms as described by the indicated equations in the text. Only the distribution of relaxation times given by Eq. (5) can account for the lowfrequency behavior at all $T$. 
tures where it is meaningful to extract all four fitting parameters, the large- $\tau$ tail diminishes with increasing temperature in both amplitude and extent (see Fig. 4), as measured by $a$ and $y$, respectively. Note that $y$, which is essentially $d \ln \chi^{\prime \prime}(\omega) / d \ln \omega$ for $10^{-3} / \tau_{c}<\omega$ $<10^{-1} / \tau_{c}$, increases as $T$ decreases. This result contrasts with spin-glass behavior where $y$ is expected ${ }^{9}$ to increase with $T$.

Good fits are obtained with $a=0$ for $T \leq 120 \mathrm{mK}$, but we find that all four parameters must be varied to account for the changing behavior between 300 and 150 $\mathrm{mK}$. For $T=150 \mathrm{mK}$, the quality of the fit is not very sensitive to the value of $a$ as long as $0.1 \lesssim a \lesssim 0.5$. Above $150 \mathrm{mK}$, the values of the fitting parameters are unique, as established by our analysis. We estimate error bars in each parameter by refitting the data with the parameter in question held fixed over a range around its best-fit value. This approach provides a measure of the curvature of the $\chi^{2}$ surface as a function of each parameter. We define the error bars as the changes in the parameters which yield a $10 \%$ change in $\chi^{2}$. Two minima were discovered in the $\chi^{2}$ surface, but we ruled out the minimum which gave an unphysical (in gross disagreement with $2 \pi / f_{\rho}$ ) value for $\tau_{c}$.

A fruitful approach to an understanding of the dynamics of disordered systems is to consider the distribution of energy barriers, $\rho_{E}$, which would lead to the distribution of relaxation times, $\rho_{\tau}$, consistent with experiment. In general, one associates a time scale $\tau\left(E_{B}\right)=\tau_{0} \exp \left(E_{B} /\right.$ $k T)$ [ $\tau_{0}$ is fixed for all $E_{B}$; see also Eq. (1)] with each

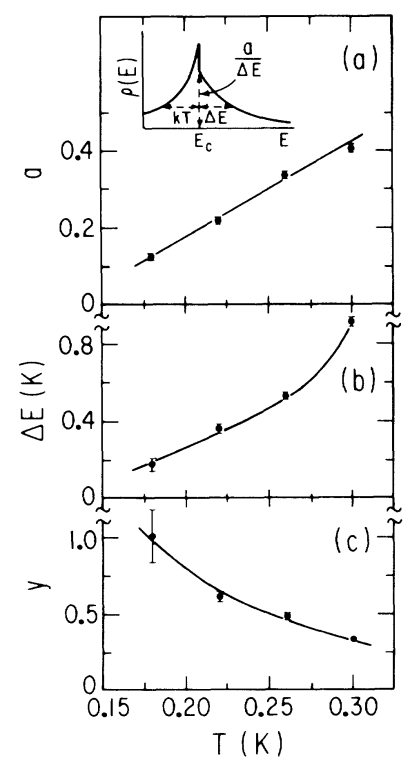

FIG. 4. Fitting parameters as functions of temperature for the distribution of energy barriers shown in the inset. The width of $\rho(E)$ actually decreases with decreasing $T$. Solid lines are guides to the eye. barrier $E_{B}$, so that

$$
\rho_{E}\left(E_{B}\right)=(k T)^{-1} \tau_{0} \exp \left(E_{B} / k T\right) \rho_{\tau}\left(\tau_{0} \exp \left(E_{B} / k T\right)\right) .
$$

For the distribution function given by $(5), \rho_{E}\left(E_{B}\right)$ is shown in the inset to Fig. 4(a). The important features are the maximum at $k T \ln \left(\tau_{c} / \tau_{0}\right)=E_{c}$, and the exponential tails on either side of $E_{c}$. The low-energy tail is proportional to $\exp \left[\left(E-E_{c}\right) / k T\right]$, while the high-energy tail falls off as $\exp \left[\left(E_{c}-E\right) / \Delta E\right]$. In the present experiment $E_{c} \gg k T$, so that the width of the low-energy tail is $k T$. The exponential tails dominate $\chi(\omega, T)$; a smoother crossover in $\rho_{E}$ at $E_{c}$ will yield a $\chi(\omega)$ insignificantly different from that given by Eqs. (4) and (5) or that observed experimentally.

The main results of our measurements can be summarized as follows: (i) The typical barrier height, $E_{c}$, is temperature independent. (ii) As $T$ is reduced, the width $\Delta E=k T / y$ of $\rho_{E}\left(E_{B}\right)$ is reduced [see Fig. 4(b)]. In particular, both the population $\left[a=\int_{E_{c}}^{\infty} \rho\left(E_{B}\right) d E_{B}\right]$ and the mean deviation $(\Delta E)$ from $E_{c}$ of barriers with $E_{B}>E_{c}$ decrease more rapidly with $T$ than $T^{1}$. The mean deviation from $E_{c}$ of barriers with $E_{c}<E_{B}$ also decreases with $T$, but only in direct proportion to $T$.

These results are unusual because almost all disordered magnets studied to date display the opposite behavior. Indeed, random magnets generally undergo freezing transitions marked by the proliferation of high barriers as $T$ is reduced. ${ }^{10}$ Similarly, the dielectric response $^{11}$ of the dipolar glass $(\mathrm{KBr})_{x}(\mathrm{KCN})_{1-x}$ is characterized by a $\chi^{\prime \prime}(\omega)$ which broadens as $T$ decreases. However, in this case, the dielectric susceptibility (which measures dipolar motion) appears to be coupled to and dominated by a quadrupolar freezing transition. $^{12}$

An explanation of our data must begin with a ground state where spin-cluster reorientation is governed by a single barrier, $E_{c}$. As the system is heated, excited spin clusters appear and raise the barriers to reorientation within spin clusters in their ground-state configuration. Such a scenario is plausible if we assume that in the ground state of $\mathrm{LiHo}_{0.045} \mathrm{Y}_{0.955} \mathrm{~F}_{4}$, the system is divided into finite spin clusters whose net dipole moments vanish. Thus, reorientation of a single cluster occurs in a molecular field which is effectively zero. On the other hand, if some clusters are in excited states, their net dipole moments need no longer vanish, and they will exert a field even on distant clusters attempting to reorient. The distribution of fields will broaden as $T$ and the population of excited clusters are increased. Consequently there will be a broadening of $\rho_{E}$. An analogous mechanism, although formulated in the language of tunneling centers, has been proposed ${ }^{13}$ for deviations from the "ideal" linear specific heat of (structurally) disordered solids.

We have presented detailed measurements of the frequency-dependent magnetic susceptibility of a dilute 
dipolar-coupled Ising magnet. In contrast both to what occurs for most random magnets ${ }^{3}$ and to the theoretical expectation of a spin-glass transition at low $T,{ }^{14}$ there is a progressive loss of high barriers to spin relaxation as $T$ is reduced. However, it is possible to interpret the results in terms of a ground state where spin clusters are decoupled from each other because they carry net zero dipole moments. Thus, at low temperatures, a dilute set of interacting dipoles behaves essentially as a set of decoupled two-level systems. This is reminiscent of an interacting Fermi liquid which, at sufficiently low $T$, will behave as a collection of independent quasiparticles.

We should like to thank R. N. Bhatt, N. O. Birge, D. Huse, S. R. Nagel, and C. M. Varma for illuminating discussions. The work at The University of Chicago was supported by the National Science Foundation Materials Research Laboratory under Grant No. 85-19460. One of us (T.F.R.) acknowledges support from the Alfred P. Sloan Foundation.

${ }^{1}$ See, for example, G. H. Wannier, Phys. Rev. 79, 357 (1950); J. Stephenson, J. Math. Phys. 11, 413 (1970).

${ }^{2}$ I. R. McLenaghan and D. Sherrington, J. Phys. C 17, 1531
(1984).

${ }^{3}$ See, for example, K. Binder and A. P. Young, Rev. Mod. Phys. 58, 801 (1986).

${ }^{4}$ D. H. Reich, T. F. Rosenbaum, G. Aeppli, and H. Guggenheim, Phys. Rev. B 34, 4956 (1986).

${ }^{5}$ For further details of the digital lock-in technique, see N. O. Birge and S. R. Nagel, Rev. Sci. Instrum. (to be published).

${ }^{6}$ A. M. Morrish, The Physical Principles of Magnetism (Wiley, New York, 1965), Appendix II.

${ }^{7}$ P. Debye, Polar Molecules (Chemical Catalogue, New York, 1929), p. 94.

${ }^{8}$ D. W. Davidson and R. H. Cole, J. Chem. Phys. 19, 1484 (1951)

${ }^{9}$ A. Ogielski, Phys. Rev. B 32, 7384 (1985). For experimental evidence of an enhanced low-frequency response in spinglasses as $T$ is reduced, see, for example, D. Hüser, L. E. Wenger, A. J. van Duyneveldt, and J. A. Mydosh, Phys. Rev. B 27, 3100 (1983); C. C. Paulsen, S. J. Williamson, and H. Maletta, Phys. Rev. Lett. 59, 128 (1987).

${ }^{10}$ D. S. Fisher, Phys. Rev. Lett. 56, 416 (1986).

${ }^{11}$ N. O. Birge, Y. H. Jeong, S. R. Nagel, S. Bhattacharya, and S. Susman, Phys. Rev. B 30, 2306 (1984).

${ }^{12}$ J. P. Sethna, S. R. Nagel, and T. V. Ramakrishnan, Phys. Rev. Lett. 53, 2489 (1984).

${ }^{13}$ C. M. Varma, R. C. Dynes, and J. R. Banavar, J. Phys. C 15, L1221 (1982).

${ }^{14}$ M. J. Stephen and A. Aharony, J. Phys. C 14, 1665 (1981). 